\section{Impact of ibrutinib dose intensity on patient outcomes in previously treated Waldenström macroglobulinemia}

In 2015, the Bruton tyrosine kinase (BTK) inhibitor ibrutinib became the first approved therapy for symptomatic Waldenström macroglobulinemia (WM). ${ }^{1}$ Temporary interruption of therapy is recommended to manage treatment-related toxicities and when patients undergo invasive procedures. $^{2}$ In a previous study, a lower dose intensity (DI) of ibrutinib therapy was associated with a shorter progression-free survival (PFS) in 195 patients with chronic lymphocytic leukemia (CLL)/small lymphocytic lymphoma (SLL) who participated in the RESONATE study. ${ }^{3}$ However, the impact of interrupted therapy with ibrutinib has not been evaluated in patients with WM. We examined the importance of ibrutinib DI in previously treated WM patients who received therapy in the multicenter study that supported regulatory approval (clinicaltrials.gov identifer: 01614821). ${ }^{4}$

Medical records of subjects who participated in this trial were reviewed and pertinent clinical data were collected surrounding ibrutinib temporary drug holds. Treatment adherence to ibrutinib was documented during the clinical trial and was measured by overall DI. Overall DI was defined as the proportion of administered versus planned daily $420 \mathrm{mg}$ doses from initiation of therapy until last dose received. We also evaluated the 8week DI and 6-month DI, which were defined as the proportion of administered versus planned daily $420 \mathrm{mg}$ doses within the first eight weeks and six months, respectively, from initiation of ibrutinib therapy. Response was assessed according to current criteria. ${ }^{5}$ Comparison between groups were evaluated using $\chi^{2}$ or Fisher exact tests. PFS was defined as the time in months from ibrutinib initiation until disease progression or death from any cause, and was estimated using the Kaplan-Meier method. Comparisons between groups were made using the log-rank test. Survival outcomes were analyzed using Cox proportional-hazard regression models and reported as Hazard Ratio (HR) with 95\% Confidence Interval (CI). Assessment of MYD88 and CXCR4 mutations were performed as previously described. ${ }^{6,7}$

Sixty-three WM patients were enrolled and began therapy with ibrutinib at $420 \mathrm{mg}$ once daily. Of these patients, $20(32 \%)$ had an overall DI of $100 \%, 37$ patients $(59 \%)$ had an overall DI of $95-99 \%$, and 6 patients $(10 \%)$ had an overall DI below $95 \%$. Only one patient $(1.6 \%)$ had an overall DI below $80 \%$. After a median of 3.9 years of ibrutinib therapy, the mean overall DI was $97 \%$. Nineteen patients $(30 \%)$ had low overall DI (mean DI $<97 \%$ ), and 44 patients $(70 \%)$ had high overall DI (mean DI $>97 \%$ ). Patients with low overall DI were more likely to be 65 years or older at the time of ibrutinib initiation compared to patients with high overall DI (68\% vs. $36 \%$; $P=0.03)$. No other differences could be demonstrated between patients with low versus high overall DI (Table 1 ). The median drug hold length was six days (range $2-50$ days). Ibrutinib DI was decreased due to toxicity in $57 \%$, and due to surgical procedures in $43 \%$ of patients.

At the time of this report, the median follow-up time is 48 months (95\% CI: 47-50 months). Twenty-four patients $(38 \%)$ have progressed, and the median PFS has not yet been reached. The median PFS was significantly shorter in patients with low overall DI versus high overall DI (median 22 months vs. not reached; log-rank $P=0.001$ ) (Figure 1A). The HR for PFS in patients with low overall DI was 3.59 (95\%CI: 1.58-8.14; $P=0.002)$ when compared with patients with high overall DI. In a multivariate analysis adjusting for age, serum IgM, hemoglobin and $\beta 2$-microglobulin levels, low DI was the only independent variable associated with worse PFS (HR 3.34, 95\% CI: 1.34-8.30; $P=0.009$ ).

Based on genomic profiling, 37 patients $(59 \%)$ were MYD $88^{\mathrm{MUT}} C X C R 4^{\mathrm{WT}}, \quad 21$ patients $(33 \%)$ were MYD $88^{\mathrm{MUT}} C X C R 4^{\mathrm{WHIM}}$, and 5 patients $(8 \%)$ were

Table 1. Patients' baseline characteristics according to ibrutinib dose intensity (DI).

\begin{tabular}{|c|c|c|c|}
\hline Characteristic & $\begin{array}{l}\text { High overall DI } \\
\qquad(n=44)\end{array}$ & $\begin{array}{c}\text { Low overall DI } \\
(n=19)\end{array}$ & $P$ \\
\hline Age $\geq 65$ years & $16(36 \%)$ & $13(68 \%)$ & 0.03 \\
\hline Male sex & $34(77 \%)$ & $14(74 \%)$ & 0.76 \\
\hline Hemoglobin $<10 \mathrm{~g} / \mathrm{dL}$ & $15(34 \%)$ & $10(53 \%)$ & 0.26 \\
\hline Platelet $<\times 10^{9} / \mathrm{L}$ & $3(7 \%)$ & $4(21 \%)$ & 0.18 \\
\hline Serum 2-microglobulin $>3.0 \mathrm{mg} / \mathrm{L}$ & $33(75 \%)$ & $11(58 \%)$ & 0.23 \\
\hline Serum $\operatorname{lgM}>4000 \mathrm{mg} / \mathrm{dL}$ & $18(41 \%)$ & $8(42 \%)$ & 1.00 \\
\hline Bone marrow involvement $\geq 50 \%$ & $27(61 \%)$ & $10(53 \%)$ & 0.58 \\
\hline Lymphadenopathy & $27(61 \%)$ & $10(53 \%)$ & 0.58 \\
\hline Splenomegaly & $3(7 \%)$ & $4(21 \%)$ & 0.18 \\
\hline Number of prior therapies $>2$ & $17(39 \%)$ & $10(53 \%)$ & 0.41 \\
\hline MYD88 L265P mutation & $40(91 \%)$ & $18(95 \%)$ & 1.00 \\
\hline CXCR4 mutation & $12(27 \%)$ & $9(47 \%)$ & 0.15 \\
\hline \multicolumn{4}{|l|}{ Best response } \\
\hline Very good partial response & $13(30 \%)$ & $5(26 \%)$ & 0.73 \\
\hline Partial response & $22(50 \%)$ & $9(47 \%)$ & \\
\hline Minor response & $6(14 \%)$ & $2(11 \%)$ & \\
\hline Stable disease & $3(7 \%)$ & $3(16 \%)$ & \\
\hline
\end{tabular}


$M Y D 88^{\mathrm{wT}} C X C R 4^{\mathrm{wT}}$. Subgroup analyses showed a significant difference in PFS for $M Y D 88^{\mathrm{MUT}} C X C R 4^{\mathrm{WT}}$ patients with low DI versus high DI [median 35 months vs. not reached (NR); $P=0.007$ ] (Figure $1 \mathrm{~B}$ ). The HR for PFS in patients with low overall DI was 4.83 (95\% CI: 1.38-16.9; $P=0.01)$ when compared with patients with high overall DI. There was a trend in $M Y D 88^{\mathrm{MUT}} C X C R 4^{\mathrm{WHIM}}$ patients (median 14 months vs. NR; log-rank $P=0.05$ ) (Figure 1C). The HR for PFS in patients with low overall DI was 3.37 (95\%CI: 0.95-12.0; $P=0.06$ ) when compared with patients with high overall DI. No difference could be demonstrated in MYD $88^{\mathrm{WT}} C X C R 4^{\mathrm{WT}}$ patients (median 5 months vs. NR; HR 1.09, 95\%CI: 0.09-13.3; P=0.95), but the sample was too small to make reliable estimates.

The mean 8-week DI was 96\% (range 61-100\%). Fifty- one patients (81\%) had an 8 -week DI more than $96 \%$, and 5 patients ( $8 \%$ ) had an 8 -week DI less than $80 \%$. The mean 6-month DI was $98 \%$ (range $84-100 \%$ ). Fiftyone patients $(81 \%)$ had a 6 -month DI more than $98 \%$, and no patients had a 6 -month DI of less than $80 \%$. The 8-week and 6-month DI below the mean were not associated with worse PFS (log-rank $P=0.75$ in both cases). We also evaluated the median DI $(99 \%)$ as a cutoff for our survival analysis; 32 patients $(51 \%)$ had a median DI more than $99 \%$, and 31 patients (49\%) had a median DI of $99 \%$ or under. There was no statistical difference in median PFS between patients with median DI more than $99 \%$ and median DI of $99 \%$ or under (NR vs. 47 months; log-rank $P=0.17)$.

Among patients who held ibrutinib ( $\mathrm{n}=50 ; 79 \%)$, those
A

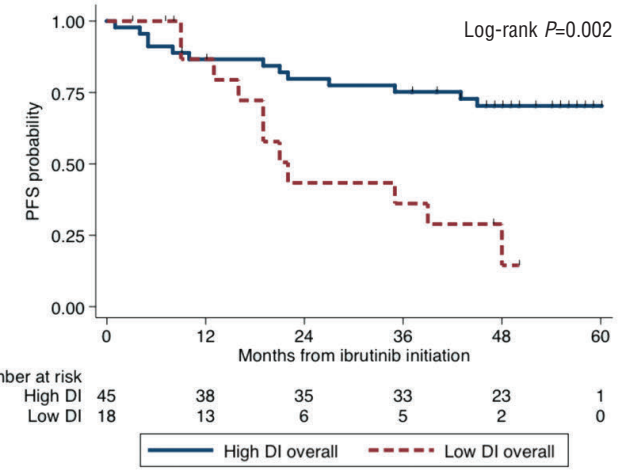

C

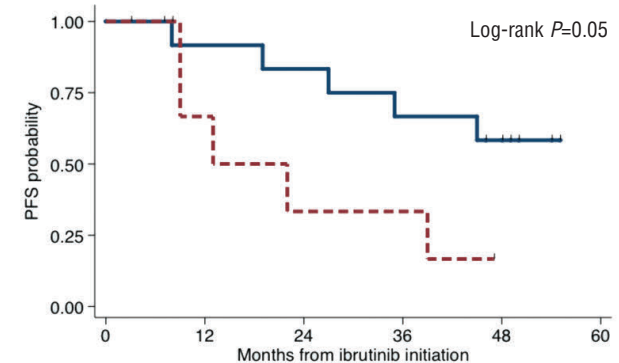

Number at risk

$\begin{array}{ll}\text { High DI } & 12 \\ \text { Low DI } & 9\end{array}$

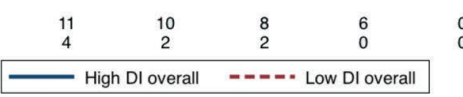

E

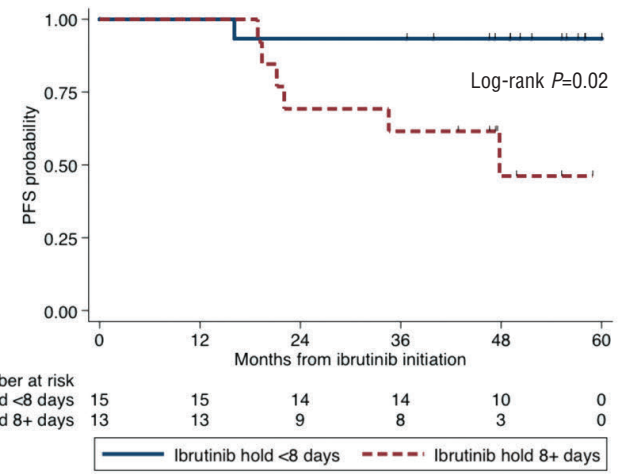

B

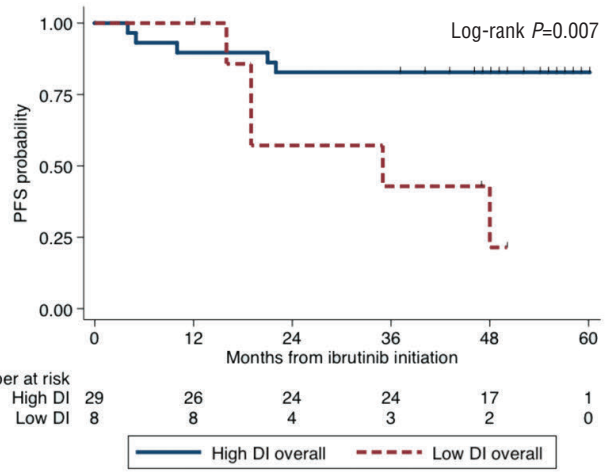

D

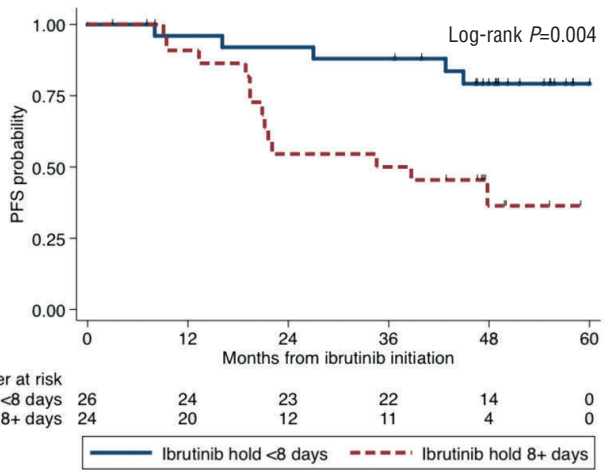

$\mathbf{F}$

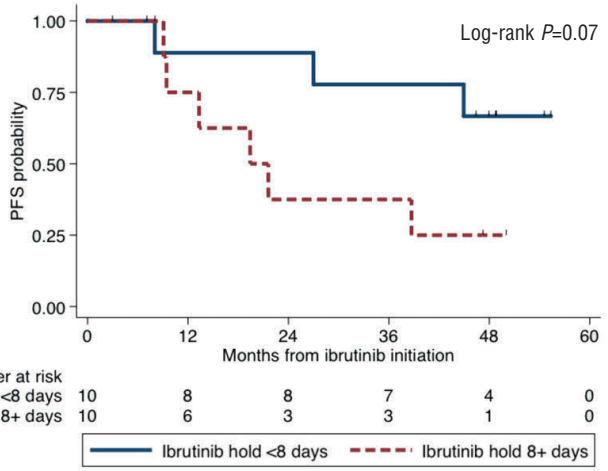

Figure 1. Progression-free survival (PFS) estimates in patients with 63 patients with relapsed and/or refractory Waldenström macroglobulinemia treated with ibrutinib. Kaplan-Meier curves are shown according to ibrutinib overall dose intensity (DI) in (A) all patients, (B) MYD88 mutated/CXCR4 wild-type patients, and (C) MYD88 mutated/CXCR4 mutated patients, and according to holding ibrutinib for eight consecutive days at any time during treatment in (D) all patients, (E) MYD88 mutated/CXCR4 wild-type patients, and (F) MYD88 mutated/CXCR4 mutated patients. 
who missed doses for eight consecutive days or more $(n=26 ; 41 \%)$ experienced a shorter PFS compared to patients who missed less than eight consecutive days $(\mathrm{n}=24 ; 38 \%)$ with a median PFS of 35 months versus NR $(P=0.005)$ (Figure 1D). The HR for PFS in patients who held ibrutinib for eight or more days was 4.07 (95\% CI: 1.44-11.5; $P=0.008$ ) when compared to patients who held for less than eight days. In $M Y D 88^{\mathrm{MUT}} C X C R 4^{\mathrm{WT}}$ patients, holding for eight days or more was associated with a median PFS of 48 months versus NR in patients who held for less than eight days (log-rank $P=0.01$ ) (Figure 1E). The HR for PFS in patients who held ibrutinib for eight or more days was 8.56 (95\%CI: 1.02-72.0; $P=0.04)$ when compared to patients who held for less than eight days. In MYD $88^{\mathrm{MUT}} C X C R 4^{\mathrm{WHIM}}$ patients, holding ibrutinib for eight days or longer was associated with a median PFS of 22 months versus NR in patients who held for less than eight days (log-rank $P=0.07$ ) (Figure $1 F$ ). The HR for PFS in patients who held ibrutinib for eight or more days was 3.49 (95\%CI: $0.85-14.4 ; P=0.08)$ when compared to patients who held for less than eight days. There was a weak positive correlation between holding ibrutinib and low DI (Pearson's r=0.24).

An increase in serum IgM level was observed on 62 occasions at the next response assessment after a drug hold. The median increase in serum IgM level was $51 \%$ (range $5-552 \%$ ), and 37 increases $(60 \%)$ met criteria for progressive disease (PD). Following the reinitiation of ibrutinib, the median time to a response of stable disease (SD) or better was 127 days, and was significantly longer for patients with the MYD $88^{\mathrm{MUT}} C X C R 4^{\mathrm{WHIM}}$ versus $M Y D 88^{\mathrm{MUT}} C X C R 4^{\mathrm{WT}}$ tumor genotype (212 vs. 109 days; $P<0.001)$.

Our retrospective analysis of this single-arm prospective study suggests that WM patients with mean overall DI lower than $97 \%$ had shorter PFS and a risk of progression 3 times higher than in patients with a mean overall DI of $97 \%$ or higher. In addition, holding ibrutinib for longer than one week at any time during the entire treatment duration appeared to be associated with a 4-fold increased risk of progression. Our stratified analyses based on tumor genotype showed similar results for overall DI and ibrutinib hold lasting longer than one week. Our findings are in line with a previous study evaluating the impact of ibrutinib dose intensity in patients with $\mathrm{CLL}^{3}{ }^{3}$ and suggest that ibrutinib holds should be minimized, and ibrutinib restarted as soon as clinically indicated to achieve optimal patient outcomes. However, low DI at eight weeks or six months did not adversely impact PFS in our cohort, when compared to a study in CLL. ${ }^{3}$ A potential explanation is the longer median PFS in patients with low DI in our cohort of 22 months versus seven months in the CLL study. Temporary interruption of ibrutinib therapy was associated with transient increases in serum IgM level, and more than half of WM patients temporarily holding ibrutinib would experience disease progression based on current criteria. ${ }^{5}$ However, a response is regained in virtually all cases at ibrutinib reinitiation, as previously reported. ${ }^{8}$ The time to response after progression during a temporary hold appears longer for WM patients with the MYD $88^{\mathrm{MUT}} C X C R 4^{\mathrm{WHIM}}$ tumor genotype.
We acknowledge the limitations of our study, which include the small sample and smaller subset analyses, which could have introduced bias into our results. One could also argue that the low DI seen in our study might not reflect a real-world experience and could make the interpretation of our results challenging. On the other hand, the problem of missing data was minimized by the prospective nature of the parent study, and the median follow-up time is the longest reported in WM patients. In conclusion, our study suggests that, similar to CLL patients, low DI adversely impacts PFS in WM patients. Ibrutinib therapy is indefinite and compliance should be strongly emphasized to optimize outcomes.

Jorge J. Castillo, ${ }^{1,2}$ Joshua N. Gustine, ${ }^{1}$ Kirsten Meid, ${ }^{1}$

Toni E. Dubeau, Lian Xu, Guang Yang, ${ }^{1,2}$

Zachary R. Hunter, Ranjana Advani, ${ }^{3}$ Lia Palomba ${ }^{4}$ and Steven P. Treon, ${ }^{1,2}$

${ }^{1}$ Bing Center for Waldenstrom Macroglobulinemia, Dana-Farber Cancer Institute, Boston, MA; ${ }^{2}$ Harvard Medical School, Boston, MA; ${ }^{3}$ Stanford University Medical Center, Stanford, CA and ${ }^{4}$ Memorial Sloan Kettering Cancer Center, New York, NY, USA

Acknowledgments: portions of this research were presented at the $59^{\text {th }}$ American Society of Hematology Annual Meeting in Atlanta, GA, USA. JJC would like to acknowledge the support of the WMR fund.

Correspondence: jorgej_castillo@dfci.harvard.edu doi:10.3324/haematol.2018.191999

Information on authorship, contributions, and financial \& other disclosures was provided by the authors and is available with the online version of this article at www. haematologica.org.

\section{References}

1. FDA News Release. FDA explands aproved use of Imbruvica for rare form of non-Hodgkin lymphoma. Available from: http:// www.fda.gov/newsevents/newsroom/pressannouncements/ucm 43 2123.htm. Last accessed 14 Dec 2017.

2. Castillo JJ, Palomba ML, Advani R, Treon SP. Ibrutinib in Waldenström macroglobulinemia: latest evidence and clinical experience. Ther Adv Hematol. 2016;7(4):179-186.

3. Barr PM, Brown JR, Hillmen P, et al. Impact of ibrutinib dose adherence on therapeutic efficacy in patients with previously treated CLL/SLL. Blood. 2017;129(19):2612-2615.

4. Treon SP, Tripsas CK, Meid K, et al. Ibrutinib in previously treated Waldenstrom's macroglobulinemia. $\mathrm{N}$ Engl J Med. 2015;372(15):1430-1440.

5. Owen RG, Kyle RA, Stone MJ, et al. Response assessment in Waldenstrom macroglobulinaemia: update from the VIth International Workshop. Br J Haematol. 2013;160(2):171-176.

6. Hunter ZR, Xu L, Yang G, et al. The genomic landscape of Waldenstrom macroglobulinemia is characterized by highly recurring MYD88 and WHIM-like CXCR4 mutations, and small somatic deletions associated with B-cell lymphomagenesis. Blood. 2014;123(11):1637-1646.

7. Xu L, Hunter ZR, Yang G, et al. MYD88 L265P in Waldenstrom macroglobulinemia, immunoglobulin $\mathrm{M}$ monoclonal gammopathy, and other B-cell lymphoproliferative disorders using conventional and quantitative allele-specific polymerase chain reaction. Blood. 2013;121(11):2051-2058

8. Gustine JN, Meid K, Dubeau T, et al. Ibrutinib discontinuation in Waldenstrom macroglobulinemia: Etiologies, outcomes, and IgM rebound. Am J Hematol. 2018;93(4):511-517. 